\title{
Influências do Diâmetro e Umidade da Madeira na Qualidade do Tratamento Preservativo
}

\author{
Dercílio Junior Verly Lopes ${ }^{1}$, Juarez Benigno Paes ${ }^{2}$, Ivaldo Pontes Jankowsky ${ }^{3}$, \\ Pedro Gutemberg de Alcântara Segundinho ${ }^{1}$, Graziela Baptista Vidaurre ${ }^{1}$ \\ ${ }^{1}$ Universidade Federal do Espirito Santo - UFES, Jerônimo Monteiro/ES, Brasil \\ ${ }^{2}$ Departamento de Ciências Florestais e da Madeira, Universidade Federal do Espírito Santo - UFES, Jerônimo \\ Monteiro/ES, Brasil \\ ${ }^{3}$ Escola Superior de Agricultura "Luiz de Queiroz", Universidade de São Paulo - USP, Piracicaba/SP, Brasil
}

\section{RESUMO}

Avaliaram-se os efeitos do teor de umidade e classe diamétrica na qualidade do tratamento de moirões de Corymbia torelliana e Eucalyptus grandis x Eucalyptus urophylla. Utilizaram-se 108 moirões, sendo 36 tratados e 18 não tratados, com 2,20 m de comprimento e umidade de 15 a 20\%; 20 a 30\%; 30 a $40 \%$, e diâmetros de 8 a $10 \mathrm{~cm} ; 10$ a $12 \mathrm{~cm} ; 12$ a $14 \mathrm{~cm}$. No tratamento, foram utilizados $2 \%$ de ingredientes ativos de CCA-C, vácuo inicial e final de $550 \mathrm{mmHg}$ (30 min), pressão de $11 \mathrm{kgf} \mathrm{cm}^{-2}(1 \mathrm{~h})$. Os moirões tiveram a umidade avaliada pelo medidor elétrico, que foi adequado para estimar a umidade destes. Obtiveram-se penetrações satisfatórias e a maior retenção foi obtida nos moirões com umidade de 15 a $20 \%\left(8,23 \mathrm{~kg} \mathrm{~m}^{-3}\right)$, contudo, umidade de 30 a $40 \%$ também foi adequada ao tratamento da madeira, obtendo valores superiores a $6,5 \mathrm{~kg} \mathrm{~m}^{-3}$.

Palavras-chave: tratamento de moirões, arseniato de cobre cromatado, penetração, retenção.

\section{Influences of Diameter and Wood Moisture on Quality of the Preservative Treatment}

\begin{abstract}
Were evaluated the effects of moisture content and diametric class in industrial treatment quality of Corymbia torelliana and Eucalyptus grandis $\mathrm{x}$ Eucalyptus urophylla fence posts. 108 fence posts were used, 36 treated and 18 non-treated, with $2.20 \mathrm{~m}$ long and moisture content of 15 to $20 \% ; 20$ to $30 \%$; and 30 to $40 \%$ and diameters of 8 to $10 \mathrm{~cm} ; 10$ to $12 \mathrm{~cm} ; 12$ to $14 \mathrm{~cm}$. For treatment, were used $2 \%$ active ingredients of CCA-C, initial and final vacuum $550 \mathrm{mmHg}$ (30min), pressure of $11 \mathrm{kgf} \mathrm{cm}^{-2}(1 \mathrm{~h})$. The fence posts were evaluated by electric meter that was adequate to estimate the moisture. Satisfactory penetrations were obtained and the biggest retention was reached in fence posts with moisture of 15 to $20 \%\left(8.23 \mathrm{~kg} \mathrm{~m}^{-3}\right)$, however, the moisture content of 30 to $40 \%$ was also suitable for the treatment of wood, getting values higher than $6.5 \mathrm{~kg} \mathrm{~m}^{-3}$.
\end{abstract}

Keywords: treatment of fence posts, chromium copper arsenate, penetration, retention. 


\section{INTRODUÇÃO}

Nas mais diversas situações em que a madeira é utilizada, faz-se necessário a determinação de índices de qualidade, a fim de ampliar o conhecimento e, por consequência, seu uso. Em virtude do elevado consumo de espécies madeireiras de durabilidade natural elevada, estas estão em processo de extinção em várias regiões. Com isto, espécies de rápido crescimento, como eucalipto e pinus, estão sendo utilizadas para suprir tais necessidades, entretanto, naturalmente, estas espécies são sujeitas a deterioração rápida e inevitável, necessitando serem submetidas ao tratamento preservativo, para prolongar sua vida útil em serviço (Paes et al., 2001).

De acordo com Hunt \& Garratt (1967), a eficiência do tratamento preservativo pode ser calculada pela determinação da retenção, penetração e distribuição do produto na madeira, as quais são influenciadas pelas variáveis do processo e tipo de tratamento efetuado.

A Norma Brasileira Regulamentadora - NBR 9480, da Associação Brasileira de Normas Técnicas - ABNT (2009), estabelece que moirões de eucalipto tratados com preservativos hidrossolúveis devem ter retenção mínima de $6,5 \mathrm{~kg}$ de ingredientes ativos por $\mathrm{m}^{3}$ de madeira, e que a penetração deve ocorre em todo o alburno e na porção penetrável do cerne. Dessa forma, a eficiência do tratamento preservativo está associada à natureza do produto químico utilizado e às características da madeira (permeabilidade, relação cerne: alburno e teor de umidade), que determinam a profundidade da camada tóxica (Torres et al., 2011). Para tratamentos com a madeira de Eucalyptus spp. em autoclaves, a NBR 9480 (ABNT, 2009) estabelece que o teor de umidade seja igual ou inferior a $35 \%$.

Além dos fatores mencionados, as dimensões (diâmetro e comprimento) e o formato das peças de madeira (roliça ou prismática) influenciam na eficiência do tratamento preservativo. Com relação ao diâmetro e teor de umidade da madeira, foi constatada maior retenção nos moirões de Eucalyptus urophylla da classe de $7 \mathrm{~cm}$ em relação aos daquelas de 9,11 e $13 \mathrm{~cm}$ (Amaral et al., 2014), e também que o aumento do tempo de secagem proporcionou incremento na retenção média destes.

Como a maioria das unidades de tratamento de madeira no Brasil não possuem plantios próprios, madeiras de eucaliptos utilizadas pelas indústrias de celulose e papel e de carvão vegetal, com as dos híbridos Eucalyptus grandis $\mathrm{x}$ Eucalyptus urophylla, E. urophylla $\mathrm{x}$ E. grandis e as de Corymbia torelliana são utilizadas como moirões de cerca. Alguns estudos destacam a qualidade dessas madeiras para esta finalidade (Brito, 2013; Brito et al., 2016).

Dessa forma, o objetivo deste estudo foi avaliar os efeitos de teores de umidade e das classes diamétricas, na qualidade do tratamento preservativo de moirões de Corymbia torelliana e Eucalyptus grandis $\mathrm{x}$ Eucalyptus urophylla (E. urograndis).

\section{MATERIAL E MÉTODOS}

\subsection{Coleta e preparo do material}

Os moirões utilizados neste trabalho foram oriundos de plantios comerciais da espécie Corymbia torelliana, propagada por semente, e do híbrido de Eucalyptus grandis $\mathrm{x}$ Eucalyptus urophylla, denominado E. urograndis, de origem clonal, provenientes de uma empresa de tratamento de madeira, localizada no município de Pinheiros, região Norte do estado do Espírito Santo. Os dados referentes aos povoamentos florestais que originaram os moirões constam da Tabela 1.

Foram utilizados no estudo 108 moirões, 54 de cada espécie, de 2,20 m de comprimento, escolhidos aleatoriamente, em três níveis de teor de umidade da madeira; 15 a 20\%; 20 a 30\%; 30 a $40 \%$, e três classes de diâmetro, compreendido entre 8 e $10 \mathrm{~cm}$, 10 e $12 \mathrm{~cm}$ e 12 e $14 \mathrm{~cm}$. No total, foram utilizados

Tabela 1. Informações sobre a idade das árvores, local de plantio e espaçamento do povoamento.

Table 1. Information about the age of trees, planting and space of forest stand.

\begin{tabular}{cccccc}
\multirow{2}{*}{ Árvores } & Idade (anos) & \multicolumn{3}{c}{ Local de Plantio } & Espaçamento do \\
\cline { 3 - 6 } & & Altitude $(\mathbf{m})$ & Latitude & Longitude & $\begin{array}{c}\text { Plantio }(\mathbf{m}) \\
\text { C. torelliana }\end{array}$ \\
\hline E. urograndis & 9 & 109 & $18^{\circ} 27^{\prime} 37^{\prime \prime} \mathrm{S}$ & $40^{\circ} 10^{\prime} 36^{\prime \prime} \mathrm{O}$ & $3,00 \times 2,00$ \\
\hline
\end{tabular}


72 moirões tratados, correspondentes aos três níveis de umidade e de diâmetro, com quatro repetições para cada nível, e 36 moirões não tratados, com as mesmas características daqueles tratados, entretanto, com duas repetições, para a determinação do teor de umidade. O experimento foi conduzido em novembro de 2013.

Os moirões foram introduzidos na autoclave industrial (1,60 m de diâmetro e 12,00 m de comprimento), e foram utilizados 2\% de ingredientes ativos de CCA tipo C, com vácuo inicial e final de $550 \mathrm{mmHg}$, durante 30 minutos, pressão de tratamento de $11 \mathrm{kgf} \mathrm{cm}^{-2}$, durante uma hora. Para os moirões tratados, o teor de umidade foi determinado pelo método do medidor elétrico de umidade do tipo resistivo, antes do tratamento preservativo, na direção longitudinal do moirão. A região em que foram realizadas as leituras de umidade coincidiu com aquelas de retirada dos discos para a análise de penetração de cobre na madeira, sendo, $0,50 \mathrm{~m}$ (zona de afloramento), 1,10 m (meio do moirão) e a $10 \mathrm{~cm}$ do topo e das amostras nos discos, 1 (periferia até $1,5 \mathrm{~cm}$ ) e 2 (de 1,5 a $3,0 \mathrm{~cm}$ ) (Figura 1 ).

Após o tratamento, os moirões foram empilhados em local seco, coberto e arejado, para fixação dos sais na madeira, durante 90 dias, e transportados para o Departamento de Ciências Florestais e da Madeira (DCFM) da Universidade Federal do Espírito Santo (UFES), situado em Jerônimo Monteiro, Espírito Santo, para a realização dos ensaios de distribuição, penetração de cobre e retenção de CCA.

Desses moirões, foi mensurada a proporção de cerne e alburno nos pontos amostrados na Figura 1 e determinou-se o volume tratável de madeira, $\mathrm{m}^{3}$. Para a determinação da retenção de CCA, efetuou-se a digestão úmida das amostras de $1,5 \times 1,0 \times 2,0 \mathrm{~cm}$ (radial $\times$ tangencial $\times$ longitudinal), apenas na região de 0,50 m (Figura 1). Para tanto, empregou-se a metodologia citada por Paes et al. (2014), que constou da determinação do volume das amostras de madeira e incineração para obtenção das cinzas e sais metálicos, a $525^{\circ} \mathrm{C}$, e posterior adição de $3 \mathrm{~mL}$ da mistura dos ácidos sulfúrico, perclórico e nítrico, todos concentrados, nas proporções de 7:2:1, às cinzas obtidas pela incineração.

Foi realizada a digestão acelerada das amostras pelo aquecimento da mistura dos ácidos e cinzas, em chapas aquecidas, até a mistura ficar límpida, e realizada a diluição das soluções ácidas com água destilada, a volumes fixos de $50 \mathrm{~mL}$, e armazenadas em frascos plásticos devidamente identificados. As amostras diametralmente opostas de cada posição radial foram digeridas e analisadas em conjunto.

A quantificação dos sais presentes na madeira foi realizada por meio de espectrofotometria de absorção atômica. Com estes valores e com os volumes das amostras de madeira, os cálculos de retenção foram efetuados de acordo com a Equação 1, citada por Paes et al. (2014).

$\mathrm{R}=\frac{\mathrm{F} \times \mathrm{L} \times \mathrm{Fd} \times 10^{-3}}{\mathrm{~V}}$

em que: $\mathrm{R}=$ Retenção do elemento na madeira $\left(\mathrm{kg}\right.$ i.a. $\left.\mathrm{m}^{-3}\right)$; $\mathrm{F}=$ Fator estequiométrico empregado para transformação dos elementos químicos para óxidos $(\mathrm{Cu} \times 1,2518=\mathrm{CuO}$; Cr x 1,923= $\mathrm{CrO}_{3}$; As x 1,5339= $\left.\mathrm{As}_{2} \mathrm{O}_{5}\right) ; \mathrm{L}=$ Leitura obtida no espectrofotômetro $\left(\mathrm{mg} \mathrm{L}^{-1}\right) ; \mathrm{Fd}=$ Fator de diluição necessário para as leituras no espectrofotômetro; $\mathrm{e} \mathrm{V}=$ Volume das amostras utilizadas nas análises $\left(\mathrm{cm}^{3}\right)$.

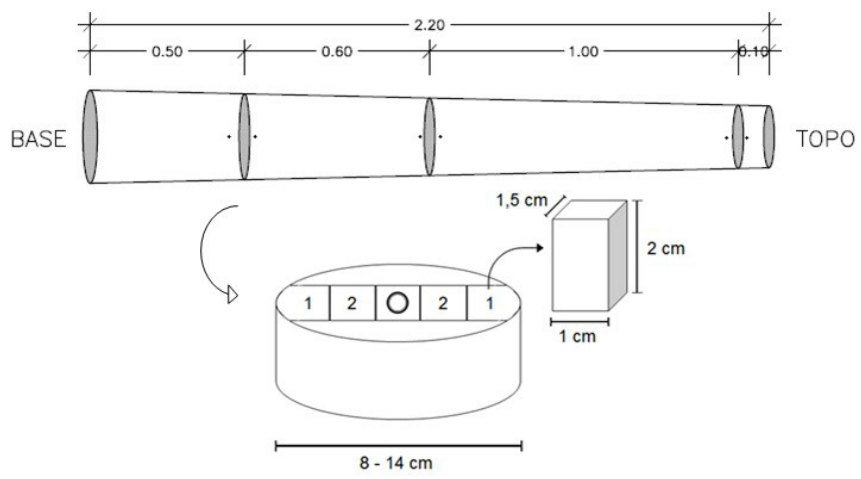

Figura 1. Locais de determinação do teor de umidade e de retirada dos discos de madeira para o desenvolvimento das análises.

Figure 1. Places of determination of moisture content and removal of disks for the development of analyses. 
A determinação da distribuição e penetração do CCA teve como base o elemento cobre presente na madeira tratada. Portanto, foi realizada a análise colorimétrica, seguindo as designações da NBR 6232 (ABNT, 2013). Os discos foram retirados em três posições: zona de afloramento $(0,50 \mathrm{~m})$; meio do moirão; a $10 \mathrm{~cm}$ do topo do moirão (Figura 1). A seção transversal dos discos foi pincelada com solução de cromo-azurol $S$, que revela uma coloração azul-escuro intensa na presença de cobre.

Para a determinação da penetração de cobre foram demarcados dois diâmetros perpendiculares entre si. O valor médio das medições foi utilizado para avaliar a penetração do elemento em cada posição nos moirões tratados, totalizando quatro medições por disco. A distribuição foi avaliada com base nas médias das medidas de penetração ao longo do comprimento de cada moirão.

Para os moirões não tratados, retiraram-se discos de $2 \mathrm{~cm}$ de espessura, na zona de afloramento $(0,50 \mathrm{~m})$, meio do moirão e a $10 \mathrm{~cm}$ do topo, para a determinação da umidade pelo método gravimétrico. Os discos foram retirados com motosserra e pesados em balança de precisão de $0,01 \mathrm{~g}$, para registrar a massa úmida, e posteriormente realizada a secagem em estufa a $103 \pm 2{ }^{\circ} \mathrm{C}$ de temperatura, até massa constante, obtendo-se a massa seca. Com base nas massas úmida e seca foi determinado o teor de umidade conforme a NBR 7190 (ABNT, 1997).

\subsection{Análise estatística dos resultados}

Para as proporções de cerne, alburno, relação cerne:alburno, volume tratável foi realizado o teste $t$ de Student para amostras independentes $(\mathrm{p} \leq 0,05)$, considerando o efeito da espécie, com base nas cinco posições longitudinais dos moirões para as três classes de diâmetro estudadas. Para a avaliação da efetividade da medição do teor de umidade, com o medidor portátil do tipo resistivo, empregou-se a análise de regressão linear simples, Pearson, modelo $(\mathrm{Y}=\mathrm{aX}+\mathrm{b})$, em que se utilizou o teor de umidade pelo método gravimétrico $(\mathrm{Y})$ em função do medidor resistivo $(\mathrm{X})$.

Uma vez que houve homogeneidade dos dados (teste de Cochran) e estes seguiram distribuição normal (teste de Lilliefors), foi realizado, para a penetração de cobre e retenção do CCA, o delineamento inteiramente casualizado com arranjo fatorial.
A penetração do elemento cobre foi avaliada para as duas espécies, em três teores de umidade, em três classes de diâmetro e em três posições do moirão (fatorial $2 \times 3 \times 3 \times 3$ ) (Figura 1). A retenção de CCA foi avaliada para duas espécies, três teores de umidade, três classes de diâmetro e duas posições radiais no disco (fatorial $2 \times 3 \times 3 \times 2$ ). Na avaliação do experimento, foi empregado o teste de Tukey $(\mathrm{p} \leq 0,05)$ para as fontes de variação detectadas como significativas pelo teste de $F(p \leq 0,05)$.

\section{RESULTADOS E DISCUSSÃO}

\subsection{Relação cerne:alburno, teor de umidade e eficácia do medidor elétrico}

Ao analisar as madeira ensaiadas, a espécie Corymbia torelliana obteve a maior porcentagem de cerne, maior relação cerne:alburno e menor volume de madeira tratável, diferindo estatisticamente do híbrido E. urograndis. Em termos de preservação de madeiras, estes fatores são primordiais na escolha da matéria-prima, haja vista que maiores porcentagens de cerne tornam a madeira menos permeável a fluidos (Tabela 2), e como o cerne formado nas árvores nas idades amostradas é proveniente de madeira juvenil, ele tem pouca resistência a organismos xilófagos (Torres et al., 2011), assim uma maior porcentagem de alburno é desejável, pois é esta a porção tratável da madeira.

A perda de permeabilidade no cerne está associada à presença de extrativos e pela formação de tiloses que obstruem o fluxo de fluido na madeira (Siau, 1995). Ressalta-se que a NBR 9480 (ABNT, 2009) regulamenta que o alburno tem de ser $100 \%$ tratável. O volume tratável de madeira maior no E. urograndis está associado à menor relação cerne:alburno.

A relação cerne:alburno afeta as características de tratabilidade (penetração e retenção do preservativo). Ao considerar o tratamento preservativo industrial, menores relações cerne:alburno seriam mais adequadas, principalmente com a utilização de árvores de idades menores, ou espécies com menores proporções de cerne.

Outro fator que influencia o tratamento industrial da madeira é o seu teor de umidade. Observa-se que a umidade que, de modo geral, melhor se ajustou para o Corymbia torelliana e E. urograndis (Figura 2) foram aquelas abaixo de $32 \%$. 
Tabela 2. Valores médios das características macroscópicas avaliadas das madeiras.

Table 2. Average values of the evaluated wood macroscopic characteristics.

\begin{tabular}{ccc} 
Características Macroscópicas & \multicolumn{2}{c}{ Madeira } \\
\cline { 2 - 3 } Avaliadas & Corymbia torelliana & E. urograndis \\
\cline { 2 - 3 } \% Cerne & $67,87 \mathrm{a}$ & $56,53 \mathrm{~b}$ \\
& $(19,74)$ & $(22,89)$ \\
\% Alburno & $32,13 \mathrm{a}$ & $43,47 \mathrm{~b}$ \\
Relação Cerne:Alburno & $(41,33)$ & $(29,74)$ \\
Volume Tratável $\left(\mathrm{m}^{3}\right)$ & $2,65 \mathrm{a}$ & $1,51 \mathrm{~b}$ \\
& $(55,47)$ & $(53,78)$ \\
\hline
\end{tabular}

Médias seguidas pela mesma letra minúscula, na horizontal, não diferem entre si pelo teste t para amostras independentes $(\mathrm{p}>0,05)$. Valor entre parênteses corresponde ao coeficiente de variação.
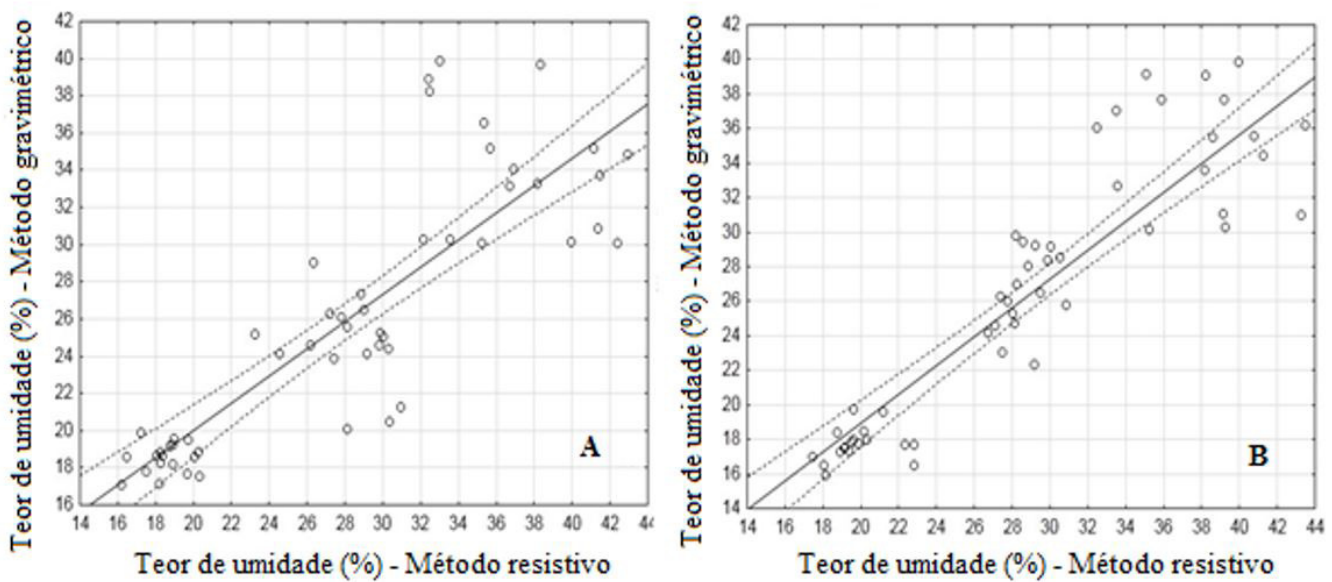

Figura 2. Regressão linear simples (Pearson) ajustada para estimar o teor de umidade da madeira de Corymbia torelliana (A) e E. urograndis (B) pelos métodos gravimétrico e resistivo. Linha contínua: linha de tendência, linha pontilhada: intervalo de confiança $95 \%$.

Figure 2. Simple linear regression (Pearson) adjusted to estimate the moisture content of the Corymbia torelliana (A) and E. urograndis (B) woods by gravimetric and resistive methods. Continuous line: line of trend, dotted line: reliable interval $95 \%$.

Alguns fatores podem ter exercido influência na medição do teor de umidade pelo medidor elétrico, destacando-se a espécie, orientação da grã, massa específica básica, temperatura local, profundidade de aplicação das agulhas do aparelho, teor de extrativos (condutividade elétrica) e constituição química da madeira (Cruz et al., 2009).

A dispersão dos dados pode ser explicada pelo intervalo em que os medidores elétricos resistivos atuam para estimar, com confiabilidade, o teor de umidade (Cruz et al., 2009). Este intervalo é compreendido entre $6 \%$ de umidade até o ponto de saturação das fibras, ou seja, valores fora deste intervalo poderão apresentar fraca correlação entre a resistência elétrica e o teor de umidade.

De acordo com a Tabela 3, a associação entre o teor de umidade pelo medidor elétrico e gravimétrico, $\mathrm{r}(\mathrm{x}, \mathrm{y})$, tanto para a espécie quanto para o híbrido, foi positiva e significativa, conforme estipulado por Larson \& Farber (2010). Para os moirões de Corymbia torelliana e E. urograndis, os modelos ajustados indicaram que o coeficiente de determinação $\left(\mathrm{R}^{2}\right)$ são adequados para estimar o teor de umidade pelo método gravimétrico.

Ressalta-se que o medidor elétrico resistivo em usinas de preservação de madeiras é uma ferramenta 
Tabela 3. Equação da reta e parâmetros estatísticos da regressão linear simples para os moirões de Corymbia torelliana e E. urograndis.

Table 3. Straight line equation and statistical parameters to simple the linear regression for Corymbia torelliana and E. urograndis fence posts.

\begin{tabular}{cccccc}
\hline Moirões & Equação da Reta de Regressão & $\mathbf{r}(\mathbf{x}, \mathbf{y})$ & $\mathbf{R}^{2}$ & Syx & $\mathbf{F}$ \\
\hline C. torelliana & $\mathrm{Y}=5,355+0,731^{\star} \mathrm{X}$ & 0,85 & 72,98 & 3,62 & $140,4^{\star *}$ \\
E. urograndis & $\mathrm{Y}=2,182+0,836^{\star} \mathrm{X}$ & 0,89 & 80,86 & 3,28 & $219,7^{\star *}$ \\
\hline
\end{tabular}

**Significativo $(\mathrm{p} \leq 0,01) ; \mathrm{r}(\mathrm{x}, \mathrm{y})$ coeficiente de correlação de Pearson; $\mathrm{R}^{2}$ coeficiente de determinação de Pearson; Syx erro padrão da estimativa.

Tabela 4. Valores médios da retenção dos ingredientes ativos de CCA em função do teor de umidade, madeira e profundidade radial.

Table 4. Average values of active ingredients retention of CCA in function on the moisture content, wood and radial depth.

\begin{tabular}{|c|c|c|}
\hline Teor de Umidade (\%) & \multicolumn{2}{|c|}{ Retenção (kg i.a m³) } \\
\hline 15 a 20 & \multicolumn{2}{|c|}{$\begin{array}{c}8,23 \mathrm{a} \\
(59,78)\end{array}$} \\
\hline 20 a 30 & \multicolumn{2}{|c|}{$\begin{array}{l}7,02 \mathrm{ab} \\
(73,93)\end{array}$} \\
\hline 30 a 40 & \multicolumn{2}{|c|}{$\begin{array}{c}6,89 \mathrm{~b} \\
(72,42)\end{array}$} \\
\hline \multirow{2}{*}{ Madeiras } & \multicolumn{2}{|c|}{ Retenção (kg i.a m ${ }^{-3}$ ) / Profundidade Radial (cm) } \\
\hline & 0 a 1,5 & 1,5 a 3,0 \\
\hline Corymbia torelliana & $\begin{array}{c}11,44 \mathrm{Aa} \\
(15,73)\end{array}$ & $\begin{array}{c}1,66 \mathrm{Bb} \\
(140,36)\end{array}$ \\
\hline E. urograndis & $\begin{array}{c}11,74 \mathrm{Aa} \\
(23,08)\end{array}$ & $\begin{array}{l}4,67 \mathrm{Ba} \\
(68,09)\end{array}$ \\
\hline
\end{tabular}

Médias seguidas pela mesma letra minúscula, na coluna, e maiúscula, na linha, não diferem entre si (Tukey, p >0,05). Valor entre parênteses corresponde ao coeficiente de variação.

de tomada de decisões importante, uma vez que esse tipo de análise é de fácil operacionalidade e de resultados rápidos, quando comparado ao método gravimétrico, o que confere agilidade na produção e evita tempo ocioso da autoclave.

\subsection{Avaliação da retenção de CCA-C na madeira}

Os resultados da análise de variância para a retenção de CCA rejeitaram a hipótese de nulidade para os efeitos dos fatores espécie, umidade e profundidade e para a interação entre espécie $\times$ profundidade, desta forma, procedeu-se a análise do teste de média (Tabela 4).

Conforme Tabela 4, ambas as espécies atingiram o mínimo de retenção de ingredientes ativos necessários para proteger a madeira, quando em contato com o solo, tendo em conta que a NBR 9480 (ABNT, 2009) regulamenta que, para madeiras a serem utilizadas nessas condições, a retenção mínima seja de $6,5 \mathrm{~kg}$ de ingredientes ativos por $\mathrm{m}^{3}$ de madeira. Portanto, os parâmetros do tratamento industrial empregados assegurariam a conformidade com a norma para ambas as madeiras contra organismos xilófagos.

Para o tratamento preservativo com CCA tipo Ca $2 \%$ de ingredientes ativos, vácuo inicial e final de $560 \mathrm{mmHg}$, por 30 e 15 minutos, respectivamente, com duração total de 90 minutos para a madeira de Eucalyptus urophylla, Valle et al. (2013) obtiveram valores de retenção superiores aos obtidos na presente pesquisa, tanto para a madeira de primeira quanto para a de segunda rotação, em que os valores médios foram de 8,85 e $8,50 \mathrm{~kg} \cdot \mathrm{m}^{-3}$, respectivamente, em virtude, principalmente, de a relação cerne:alburno ser menor que a das madeiras estudadas, em decorrência, provavelmente, da idade do material, que no caso citado foi de 63 meses (5 anos e 3 meses).

Ainda ao analisar a Tabela 4, observa-se a redução dos valores médios de retenção com o aumento do teor de umidade, ressalta-se que, acima do ponto de saturação das fibras, ocorre a diluição da solução, 
proporcionando uma maior penetração, ocasionada pelo aumento na mobilidade das moléculas de cobre e, consequentemente, menor retenção da solução preservativa na madeira. Contudo, para todos os três intervalos de umidade, a retenção foi satisfatória no que tange à indicação da NBR 9480 (ABNT, 2009).

Nota-se ainda que os maiores valores médios absolutos foram constatados na profundidade radial de 0 a $1,5 \mathrm{~cm}$ e que não houve diferença estatística entre as espécies empregadas para esta profundidade. O maior valor absoluto foi registrado na madeira de E. urograndis, para ambas as profundidades radiais.

\subsection{Avaliação qualitativa da penetração de cobre}

A penetração profunda foi indicada pela coloração azul intensa, desde a periferia da região do alburno até a de transição entre cerne e alburno. No entanto, alguns defeitos da madeira, como rachaduras de secagem, nós e, provavelmente, lenho de reação (Figura 3) influenciaram a penetração do cobre nos moirões.

Ressalta-se que regiões de defeitos, como nós, em todos os discos amostrados, não apresentaram penetração na extensão da seção do alburno. Resultado semelhante foi observado (Amaral, 2012; Amaral et al., 2014) para moirões de Eucalyptus urophylla, provenientes de árvores com 9 anos de idade.

Em casos específicos de rachaduras ao longo dos raios, ocorreu a penetração superficial do cerne,

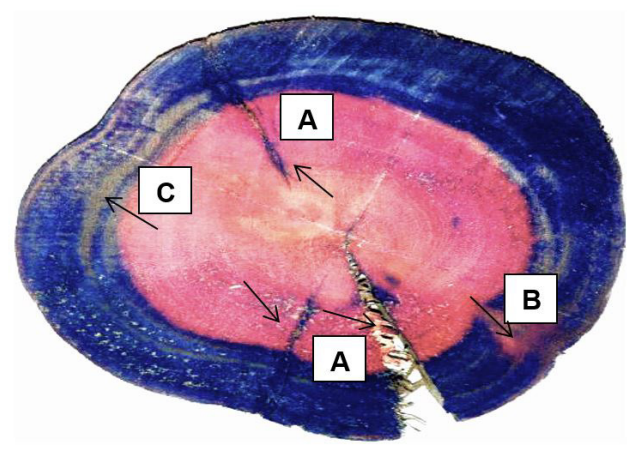

Figura 3. Penetração avaliada pela aplicação de cromoazurol-S e defeitos na madeira tratada. (A) rachaduras, (B) nó e (C) lenho de reação.

Figure 3. Penetration evaluated for chromo azurol-S application and defects in the treated wood. (A) Cracks, (B) knot and (C) reaction wood. indicando que o efeito destas pode ser comparado ao da aplicação da técnica de incisão, que causa aumento na penetração de substâncias químicas na madeira e melhora a permeabilidade do cerne (Lebow et al., 2010).

Obteve-se uma penetração satisfatória em toda região do alburno tratado, como regulamenta a NBR 6232 (ABNT, 2013). Madeiras susceptíveis ao recebimento de soluções preservativas, em função da alta permeabilidade do alburno, são descritas como não refratárias (Valle et al., 2013), denominação aplicável à espécie e híbrido estudados neste trabalho.

A penetração total do alburno confere à madeira uma elevada durabilidade contra agentes xilófagos, sendo considerada um parâmetro relevante na escolha da espécie florestal para o tratamento preservativo industrial, uma vez que madeiras menos permeáveis podem exigir maiores tempos e pressões de tratamento, aumentando o custo do processo.

\subsection{Avaliação quantitativa da penetração de cobre}

Para a penetração de cobre na madeira tratada, foram significativos os efeitos das madeiras, teor de umidade, classe de diâmetro, posição e as interações entre madeiras $\times$ umidade, madeiras $\times$ diâmetro, umidade $\times$ diâmetro, diâmetro $\times$ posição, e madeiras $\times$ umidade $\times$ diâmetro.

De acordo com a Tabela 5, ao analisar a interação entre madeiras e umidade, pode-se observar que, para o teor de umidade de 15 a $20 \%$, foram obtidas as maiores penetrações e que estas ocorreram para o E. urograndis. Destaca-se nesta mesma interação uma controvérsia em relação à penetração na umidade compreendida entre 30 e $40 \%$, em que se obteve penetração maior que para a umidade no intervalo 20 a $30 \%$, para ambas as madeiras.

Acima do ponto de saturação das fibras, o aumento da umidade dilui a solução preservativa, proporcionando uma maior penetração e, consequentemente, uma menor retenção na madeira (Lepage, 1986). Isto se deve, provavelmente, aos moirões amostrados na umidade de 30 a $40 \%$ serem provenientes de partes superiores do tronco ou com maior porcentagem de alburno (Figura 4).

Ainda ao analisar a Tabela 5, na interação entre madeira e diâmetro, nota-se que as maiores penetrações 
Tabela 5. Valores médios da penetração radial de cobre em função da madeira, do teor de umidade e classe diamétrica.

Table 5. Average values of copper radial penetration in function of wood, moisture content and diametric class.

\begin{tabular}{|c|c|c|c|}
\hline \multirow{2}{*}{ Madeira } & \multicolumn{3}{|c|}{ Penetração Radial (mm) / Teor de Umidade (\%) } \\
\hline & 15 a 20 & 20 a 30 & 30 a 40 \\
\hline C. torelliana & $\begin{array}{c}17,87 \mathrm{Ab} \\
(29,26)\end{array}$ & $\begin{array}{c}14,41 \mathrm{Cb} \\
(25,05)\end{array}$ & $\begin{array}{c}16,25 \mathrm{Bb} \\
(38,83)\end{array}$ \\
\hline E. urograndis & $\begin{array}{c}21,73 \mathrm{Aa} \\
(20,80)\end{array}$ & $\begin{array}{c}20,79 \mathrm{Ba} \\
(23,28)\end{array}$ & $\begin{array}{c}21,19 \mathrm{Aa} \\
(22,44)\end{array}$ \\
\hline \multirow{2}{*}{ Madeira } & \multicolumn{3}{|c|}{ Penetração Radial (mm) / Classe Diamétrica (cm) } \\
\hline & 8 a 10 & 10 a 12 & 12 a 14 \\
\hline C. torelliana & $\begin{array}{c}18,65 \mathrm{Ab} \\
(35,49)\end{array}$ & $\begin{array}{c}16,45 \mathrm{Bb} \\
(28,14)\end{array}$ & $\begin{array}{c}13,42 \mathrm{Ca} \\
(18,37)\end{array}$ \\
\hline E. urograndis & $\begin{array}{c}22,47 \mathrm{Aa} \\
(20,73)\end{array}$ & $\begin{array}{c}19,80 \mathrm{Ba} \\
(25,30)\end{array}$ & $\begin{array}{c}12,43 \mathrm{Ca} \\
(18,18)\end{array}$ \\
\hline
\end{tabular}

Médias seguidas pela mesma letra minúscula, na coluna, e maiúscula, na linha, não diferem entre si (Tukey, p >0,05). Valor entre parênteses corresponde ao coeficiente de variação.

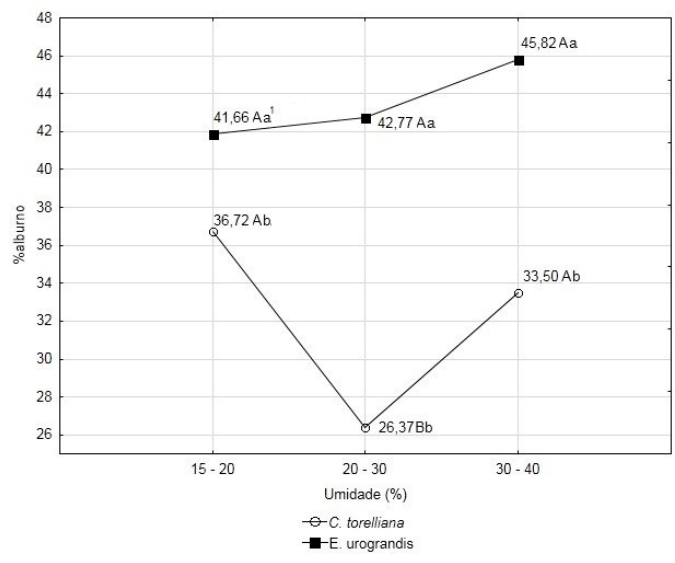

Figura 4. Porcentagem de alburno para teor de umidade pelo método resistivo para as madeiras estudadas (C. torelliana e E. urograndis). Médias seguidas pela mesma letra minúscula, entre espécie e híbrido, e por letra maiúscula na mesma espécie e híbrido, não diferem entre si, pelo teste de Tukey $(p>0,05)$.

Figure 4. Sapwood percentage for moisture content for resistive method in to the studied woods (C. torelliana and E. urograndis). Medium followed by the same lowercase letter, among species and hybrid, and uppercase letter in the same species and hybrid, not differ by Tukey's test ( $\mathrm{p}>0.05)$.

foram observadas nos menores diâmetros, com diminuição desta característica de tratabilidade concomitantemente com o aumento do diâmetro. A porcentagem de alburno possivelmente contribuiu para este comportamento da penetração (Figura 4). Com isto, ressalta-se a importância da avaliação deste parâmetro dendrométrico, pois maiores diâmetros corresponderam também à maior proporção de cerne, o qual é de madeira juvenil, sendo pouco resistente à deterioração biológica (Paes \& Vital, 2000), e isto pode desclassificar a madeira quanto ao seu uso, principalmente em contato com o solo.

As maiores médias de penetração ocorreram na classe diamétrica de 8 a $10 \mathrm{~cm}$, não diferindo estatisticamente da classe diamétrica de 10 a $12 \mathrm{~cm}$ e o teor de umidade compreendido entre 15 e 20\% (Tabela 6). O menor valor médio da penetração ocorreu na classe diamétrica de 10 a $12 \mathrm{~cm}$ no teor de umidade de 20 a 30\%, contudo, não ocorreu diferença com a classe diamétrica de 12 a $14 \mathrm{~cm}$. As penetrações no diâmetro de 12 a $14 \mathrm{~cm}$ não diferiram entre si ao analisar as classes de umidade empregadas neste trabalho.

Assim, ao considerar que uma penetração mínima satisfatória seja de 1,0 cm (Paes et al., 2005; Torres et al., 2011), o tratamento preservativo para a espécie de Corymbia torelliana e o híbrido E. urograndis foi satisfatório para o teor de umidade de até $40 \%$. $\mathrm{A}$ interação entre madeiras $\mathrm{x}$ umidade $\mathrm{x}$ diâmetro foi significativa pelo teste $F(p \leq 0,05)$ e analisada graficamente (Figura 5).

Nota-se na Figura 5 que, independente do teor de umidade e classe diamétrica, a madeira de E. urograndis atingiu os maiores valores médios de penetração de cobre, provavelmente em função do maior volume tratável de madeira. A madeira de Corymbia torelliana na classe diamétrica de 12 a $14 \mathrm{~cm}$ obteve os menores valores médios da penetração, 
Tabela 6. Valores médios de penetração para teor de umidade e a classe diamétrica.

Table 6. Average values of penetration to moisture content and the diametric class.

\begin{tabular}{|c|c|c|c|}
\hline \multirow{2}{*}{ Teor de Umidade (\%) } & \multicolumn{3}{|c|}{ Penetração Radial (mm) / Classe Diamétrica (cm) } \\
\hline & 8 a 10 & 10 a 12 & 12 a 14 \\
\hline 15 a 20 & $\begin{array}{c}19,95 \mathrm{Aa} \\
(26,06)\end{array}$ & $\begin{array}{c}21,02 \mathrm{Aa} \\
(21,5)\end{array}$ & $\begin{array}{c}18,42 \mathrm{Ba} \\
(30,89)\end{array}$ \\
\hline 20 a 30 & $\begin{array}{c}20,29 \mathrm{Aa} \\
(27,89)\end{array}$ & $\begin{array}{c}15,42 \mathrm{Bc} \\
(25,94)\end{array}$ & $\begin{array}{c}17,08 \mathrm{Ba} \\
(29,44)\end{array}$ \\
\hline 30 a 40 & $\begin{array}{c}21,44 \mathrm{Aa} \\
(32,83)\end{array}$ & $\begin{array}{c}17,95 \mathrm{Bb} \\
(28,57)\end{array}$ & $\begin{array}{c}18,27 \mathrm{Ba} \\
(34,92)\end{array}$ \\
\hline
\end{tabular}

Médias seguidas pela mesma letra minúscula, na coluna, e maiúscula, na linha, não diferem entre si (Tukey, p > 0,05). Valor entre parênteses corresponde ao coeficiente de variação.

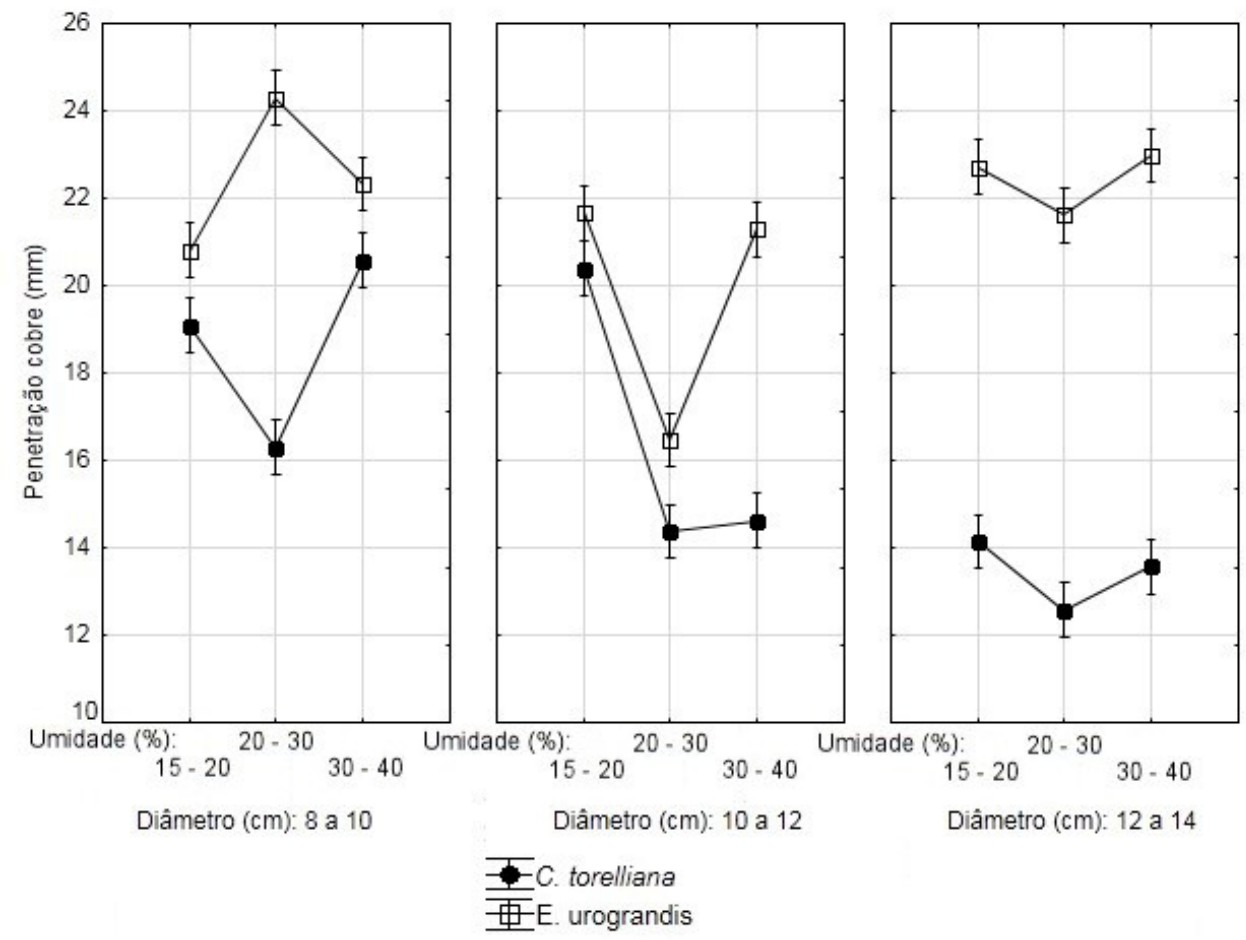

Figura 5. Análise gráfica da interação entre madeira (C. torelliana e E. urograndis), umidade e classe diamêtrica. Figure 5. Graphical analysis of interaction among wood (C. torelliana and E. urograndis), moisture and diametric class.

porém, atingiu os valores mínimos recomendáveis de $10 \mathrm{~mm}$ (Paes et al., 2005; Torres et al., 2011), para todos os intervalos de umidade.

O maior valor médio absoluto da penetração foi registrado na classe diamétrica de 8 a $10 \mathrm{~cm}$ na madeira de E. urograndis, para o intervalo de teor de umidade compreendido entre 20 e $30 \%$. Nota-se que a penetração de cobre nos diâmetros de 10 a $12 \mathrm{~cm}$ e 12 a $14 \mathrm{~cm}$ segue um padrão de diminuição, de acordo com o teor de umidade (15 a 20\%) e posterior aumento (30 a 40\%). Para o diâmetro de 8 a $10 \mathrm{~cm}$, as madeiras não tiveram padrão definido de penetração de cobre em função dos teores de umidade. Isto ocorreu em decorrência da maior quantidade de alburno nos moirões, quando comparado com as demais classes diamétricas avaliadas.

\section{CONCLUSÕES}

A medição do teor de umidade pelo método resistivo (medidor portátil de umidade) foi adequada para estimar o teor de umidade dos moirões. 
A relação cerne:alburno exerceu influência nas propriedades de tratabilidade da madeira no sentido inversamente proporcional, ou seja, quanto menor esta relação, melhores características de tratabilidade serão alcançadas.

O teor de umidade de 15 a $20 \%$ proporcionou os maiores valores médios de retenção dos ingredientes ativos do CCA para ambas as madeiras estudadas.

De maneira geral, ambas as madeiras utilizadas apresentaram penetração profunda, independente do teor de umidade e classe diamétrica.

Os parâmetros utilizados no tratamento preservativo industrial com CCA foram capazes de proporcionar aos moirões de Corymbia torelliana e E. urograndis a retenção mínima recomendada pelas normas técnicas, para as faixas de umidade e classes diamétrica avaliadas, podendo, portanto, serem utilizados em contato com solo.

\section{STATUS DA SUBMISSÃO}

Recebido: 6 maio, 2016

Aceito: 4 nov., 2016

\section{AUTOR(ES) PARA CORRESPONDÊNCIA}

\section{Juarez Benigno Paes}

Departamento de Ciências Florestais e da Madeira, Universidade Federal do Espírito Santo - UFES, Av. Governador Lindemberg, 316, CEP 29550-000, Jerônimo Monteiro, ES, Brasil e-mail: jbp2@uol.com.br

\section{REFERENNCIAS}

Amaral LS, Silva JRM, Hein PRG, Trugilho PF. Influência do diâmetro e umidade no tratamento preservativo de moirões de Eucalyptus. Revista Árvore 2014; 38(5): 919-925. http://dx.doi.org/10.1590/S0100-67622014000500016.

Amaral LS. Penetração e retenção do preservante em Eucalyptus com diferentes diâmetros [dissertação]. Lavras: Universidade Federal de Lavras; 2012. 82 p.

Associação Brasileira de Normas Técnicas - ABNT. NBR 7190: projeto de estruturas de madeira. Anexo B: determinação das propriedades das madeiras para projetos de estruturas. Rio de Janeiro; 1997. 107 p.

Associação Brasileira de Normas Técnicas - ABNT. NBR 9480: peças roliças preservadas de eucalipto para construções rurais: requisitos. Rio de Janeiro; 2009. 15 p.

Associação Brasileira de Normas Técnicas - ABNT. NBR 6232: penetração e retenção de preservativos em postes de madeira. Rio de Janeiro; 2013. 12 p.
Brito AF, Severo ET, Calonego FW. Caracterização das rachaduras em mourões de oito espécies de eucalipto. Energia na Agricultura 2016; 31(1): 59-63. http://dx.doi. org/10.17224/EnergAgric.2016v31n1p59-63.

Brito AF. Avaliação da qualidade de mourões de várias espécies de Eucalyptus spp. tratados pelo método de substituição de seiva [dissertação]. Botucatu: Faculdade de Ciências Agronômicas de Botucatu, Universidade Estadual Paulista "Julio de Mesquita Filho"; 2013. 74 p.

Cruz CR, Muniz GIB, Lima JT, Ferreira DF. Application of stress waves to estimate moisture content in Eucalyptus wood. Cerne 2009; 15(4): 430-438.

Hunt GM, Garratt GA. Wood preservation. 3. ed. New York: McGraw Hill; 1967. 433 p.

Larson R, Farber B. Estatística aplicada. 4. ed. São Paulo: Pearson Prentice Hall; 2010. 640 p.

Lebow P, Lebow S, Nelson W. Effect of treatment pressure on treatment quality and bending properties of red pine lumber. Forest Products Journal 2010; 60(5): 447-452. http://dx.doi.org/10.13073/0015-7473-60.5.447.

Lepage ES. Preservativos e sistemas preservativos. In: Lepage ES, editor. Manual de preservação de madeiras. São Paulo: IPT; 1986. p. 279-342. vol. 1.

Paes JB, Lopes DJV, Gonçalves FG, Brito FMS, Lombardi LR. Efeito da concentração na ascensão de soluções preservativas preparadas com CCB em moirões de Eucalyptus. Floresta e Ambiente 2014; 21(3): 384-393. http://dx.doi.org/10.1590/2179-8087.036913

Paes JB, Moreschi JC, Lelles JG. Tratamento preservativo de moirões de bracatinga (Mimosa scabrella Benth.) e de Eucalyptus viminalis Lab. pelo método de imersão prolongada. Cerne 2001; 7(2): 65-80.

Paes JB, Moreschi JC, Lelles JG. Avaliação do tratamento preservativo de moirões de Eucalyptus viminalis Lab. e de bracatinga (Mimosa scabrella Benth.) pelo método de substituição da seiva. Ciência Florestal 2005; 15(1): 75-86. http://dx.doi.org/10.5902/198050981825.

Paes JB, Vital BR. Resistência natural da madeira de cinco espécies de eucalipto a cupins subterrâneos, em teste de laboratório. Revista Árvore 2000; 24(1): 97-104.

Siau JF. Wood: influence of moisture on physical properties. Virginia: Polytech. Inst. Dep. of Wood Science and Forest Product; 1995. 227 p.

Torres PMA, Paes JB, Lira JA Fo, Nascimento JWB. Tratamento preservativo da madeira juvenil de Eucalyptus camaldulensis Dehnh. pelo método de substituição de seiva. Cerne 2011; 17(2): 275-282. http://dx.doi.org/10.1590/ S0104-77602011000200016.

Valle MLA, Silva JC, Della Lucia RM, Evangelista WV. Retenção e penetração de CCA em madeira de primeira e segunda rotação de Eucalyptus urophylla S. T. Blake. Ciência Florestal 2013; 23(2): 481-490. http://dx.doi. org/10.5902/198050989292. 